\title{
Pour la compétence lexicale en immersion française : la construction d'une expérimentation réussie
}

\author{
Karen Spracklin \\ Université de Moncton, Nouveau-Brunswick, Canada \\ karen.spracklin@umoncton.ca
}

\begin{abstract}
Résumé Le présent article décrit un programme d'enseignement explicite du vocabulaire mis en place et expérimenté dans des classes d'écoles secondaires offrant le programme d'immersion en français au Canada. Fondée sur l'approche actancielle de J. Picoche (1992), cette recherche expérimentale a visé l'élaboration d'un programme qui permettait l'enrichissement du vocabulaire chez l'apprenant anglophone ainsi que le développement d'une conscience du fonctionnement des mots dans la phrase. L'objectif de la partie de l'expérimentation ici décrite était de comprendre les effets de l'approche actancielle sur le développement de la compétence lexicale des participants par l'enseignement explicite des mots autour du verbe DIRE.
\end{abstract}

\begin{abstract}
Summary Canadian French Immersion classrooms served as the laboratory for an experiment in the targeted teaching of vocabulary, the description of which is presented in this article. The pedagogical program was modeled on J. Picoche's actancielle approach (1992) and sought to understand if Anglophone students' lexical development would be enhanced by an explicit instructional program concerning the role of the new words within the phrase. Vocabulary lessons involving the verb DIRE and the significance of their effects are discussed.
\end{abstract}

\section{Introduction}

L'importance du vocabulaire dans l'acquisition ou dans l'apprentissage d'une langue ne peut être sous-estimée. En effet, le vocabulaire est la clé du développement de la compétence langagière chez l'apprenant d'une langue seconde (Harley et Jean 1999). Plus précisément, la compétence lexicale est développée en travaillant de multiples facettes linguistiques des mots, qu'elles soient phonémiques, morphologiques, syntaxiques ou sémantiques (Tréville et Duquette 1996). À la base de toute formation lexicale complète se situent nécessairement l'apprentissage de la forme et du sens des mots ainsi que du rôle de ceux-ci dans la phrase. Les enjeux liés à leur maîtrise sont fondamentaux, puisqu'un manque de vocabulaire chez les apprenants d'une langue seconde constitue un sérieux obstacle à la compréhension et à la production de la langue, qu'elle soit parlée ou écrite (Jared 2008; Coady 1997). Promouvoir l'apprentissage des mots est alors impératif.

Nous décrivons ici les résultats d'une démarche expérimentale au cours de laquelle nous avons élaboré des outils pédagogiques pour l'enseignement explicite du vocabulaire français auprès des élèves anglophones du secondaire au Nouveau-Brunswick (Canada). Ce sont les piètres résultats de ces élèves dans les épreuves en écriture, et surtout au niveau du choix des mots et de la structuration de la phrase française, qui nous ont motivée à agir. 
Nous avons également constaté d'importantes lacunes sur le plan pédagogique, d'abord l'absence de matériel axé sur l'acquisition du vocabulaire et destiné aux enseignants, mais aussi le peu d'importance accordé à l'enseignement explicite des mots dans le contexte des approches communicatives actuellement en vigueur. Pourtant, ce sont les erreurs de vocabulaire - le choix impropre d'un mot - qui empêchent la communication efficace (Folse 2004; Tréville 1987). Les méthodes traditionnelles d'apprentissage du vocabulaire, qui prennent trop souvent la forme de mémorisation de longues listes de mots, ne conduisent pas à l'intégration réussie des nouveaux mots. Un enseignement systématique et ordonné du vocabulaire, fondé sur une théorie linguistique solide, s'impose.

Le programme d'enseignement que nous avons développé est fondé sur l'approche actancielle de J. Picoche (1992). Celle-ci a créé une approche cohérente de l'enseignement du vocabulaire en milieu scolaire. Dans l'approche actancielle, on cherche à développer chez l'apprenant une conscience du fonctionnement des mots dans la phrase en travaillant les rapports sémantiques des mots de la langue sur l'axe paradigmatique par le biais de l'étude des synonymes, des antonymes et des mots polysémiques, notamment, et sur l'axe syntagmatique, en appréciant la compatibilité de sens entre les mots qui constituent la phrase. Le mot n'est pas une entité indépendante; il prend son sens dans le contexte de la phrase dans laquelle il se trouve. L'approche actancielle intègre l'enseignement syntaxique à l'enseignement lexical. Cette approche novatrice a été conçue à l'origine pour l'enrichissement du lexique des élèves de français langue maternelle. Pourtant bien reconnue dans le milieu de la didactique du français, elle n'a pas fait, à notre connaissance, l'objet d'une évaluation formelle. Cet article présente la mise en œuvre de l'approche actancielle dans un contexte nouveau.

Notre texte est organisé en sept parties : la première fournit une définition opératoire de la compétence lexicale, alors que la deuxième présente l'état des lieux du programme immersif sur le terrain. Nous y expliquons les faiblesses lexicales des élèves en immersion, faiblesses qui pourraient être compensées par l'adoption d'un programme d'enseignement explicite du vocabulaire. La troisième partie est consacrée au fondement même de notre expérimentation, l'approche actancielle, qui promeut l'enseignement explicite du vocabulaire en favorisant l'intégration de la syntaxe à l'étude approfondie des mots. Précisons en outre que cette approche est très bien outillée en termes d'ouvrages lexicopédagogiques abordables qui alimenteront les leçons présentées aux élèves immersifs. Ensuite, dans la partie 4, nous expliquons le dispositif du développement des outils d'enseignement, notamment le choix des mots à enseigner, les contenus linguistiques et lexicaux à valoriser, les matériels supports nécessaires et la structuration des leçons de vocabulaire. Nous décrivons aussi l'outil développé pour l'évaluation de l'apprentissage des mots, à savoir le test de vocabulaire. Enfin, la dernière partie de l'article propose un retour sur les résultats globaux du test pour évaluer les effets linguistiques du programme d'enseignement sur l'apprentissage lexical du groupe expérimental. L'ensemble est clos par une discussion sur les effets du programme selon le point de vue des participants.

\section{Vers une définition de la compétence lexicale}

La compétence lexicale est « la capacité de reconnaître et d'utiliser les mots d'une langue comme le font les locuteurs natifs de cette langue » (SIL 1998, traduit de l'anglais). Cette capacité comprend une conscience des mots ainsi qu'une connaissance des rapports entre les familles de mots et des collocations usuelles de mots (ibid.). Robinson (1989:275) appelle ces volets de la compétence lexicale "savoir que » et "savoir comment". Autrement dit, l'apprenant doit savoir la (ou les) signification(s) du mot et savoir l'employer dans la construction des phrases, s'alignant alors avec des dimensions du savoir 
dites déclarative et procédurale dans le domaine de la linguistique cognitive. En résumé, «[c]'est la connaissance du vocabulaire et la capacité de l'utiliser. » (Cadre 2001 : 4.7.2.1.1).

L'élève doté d'une compétence lexicale jouit d'une richesse lexicale lui permettant une écriture et une expression orale plus aisées. Selon Read (2000), les mesures de richesse lexicale dans l'écriture des élèves incluent au moins quatre facettes, soit la variation lexicale c'est-à-dire l'emploi juste des synonymes et des hyperonymes, notamment; la sophistication lexicale signalée par l'emploi de mots rares ou de basse fréquence; la densité lexicale attestée par l'emploi de mots lexicaux, des noms, des verbes, des adjectifs (comparés aux mots grammaticaux tels que des prépositions, des conjonctions et des adverbes ; voir Ure 1971) et le nombre réduit d'erreurs lexicales, ce qui représente une conscience des conventions orthographiques, syntaxiques et sémantiques des mots ainsi qu'un emploi juste, précis et approprié.

Certains chercheurs estiment que la taille du lexique est moins importante que son organisation dans l'esprit de l'apprenant (Tremblay 2009:116). Autrement dit, un apprenant doté d'un lexique moyennement étendu, mais qui sait accéder dans son « lexique mental » aux mots et aux combinatoires recherchés et qui peut appliquer ces mots dans une situation de communication, aura un net avantage sur celui qui dispose d'un lexique plus impressionnant de mots isolés, mais qui ne sait pas les appliquer. Le développement de la compétence lexicale en L1 (langue maternelle) et en L2 (langue seconde) constitue une activité cumulative (Ellis 2012:100), passant de la réception d'un mot (c'est-à-dire la compréhension du mot entendu ou lu) à la production de celui-ci, et de la connaissance partielle d'un mot à la connaissance précise de celui-ci (Schmitt 1998; Jiang 2000 ; Henriksen et Haastrup 1998, cités dans R. Ellis 2012 : 100). Pour un apprenant d'une L2, ce processus graduel a une influence importante sur les productions parlée et écrite. Il incombe alors aux enseignants de langue de comprendre la nature de la compétence lexicale. Comme le reconnaît Tremblay, «si l'enseignant connaît, d'un point de vue théorique, les facettes de la compétence lexicale, il pourra mieux cibler celles qui devront être développées chez ses élèves » (2009: 108).

\section{Mise en contexte de l'étude}

Notre étude a eu lieu dans des classes d'immersion française (désormais IF dans le texte). L'IF se situe dans les approches dites communicatives. Au pluriel, le terme approches communicatives renvoie à toutes les méthodes qui ont pour but la compétence communicative, lesquelles comprennent l'approche naturelle, par exemple, et l'approche coopérative. Employée comme terme générique, l'approche communicative est aujourd'hui la référence incontournable de tous les mouvements en enseignement des langues. Elle est considérée comme étant le modèle idéal pour mener l'apprenant à une compétence linguistique imitant de plus près celle du locuteur natif.

Le programme d'IF vise l'enseignement du français aux élèves anglophones ou allophones. La compétence linguistique en français prônée par les programmes immersifs est particulièrement importante en raison du contexte sociolinguistique de notre terrain bilingue anglais-français (deux tiers de la population s'identifiant comme anglophones, et un tiers, francophones). En tenant compte de cette réalité géolinguistique et dans la volonté de promouvoir l'apprentissage de la langue française, les districts scolaires anglophones offrent des programmes d'IF qui ont pour but le développement des compétences linguistiques des élèves anglophones dans la langue de la minorité linguistique. En IF, les matières scolaires sont enseignées en français. Au programme d'IF précoce, les élèves commencent l'étude de la L2 en $3^{\mathrm{e}}$ année, c'est-à-dire à l'âge de 8 ans; dans le programme 
IF tardif, l'apprentissage commence normalement vers la $6^{\mathrm{e}}$ année scolaire, les élèves sont alors âgés de 11 ans.

Dans un programme d'IF, la langue est à la fois le moyen d'enseignement et l'objet d'apprentissage (Turnbull, Lapkin et Hart 1998), surtout dans les années scolaires précoces. Les matières scolaires traditionnelles - mathématiques, sciences, histoire, etc. - sont transmises en français, mais l'objectif des cours n'est pas explicitement l'étude de la L2. Les éléments purement linguistiques de la langue - orthographe, syntaxe et phonologie font normalement l'objet d'étude implicite grâce à l'étude de textes de lecture, à la production de travaux écrits, à des débats, etc., c'est-à-dire quand le besoin de s'arrêter sur un mot ou sur une tournure est constaté. L'IF au Canada est «basée sur le contenu» («content-based instruction ») où l'attention est portée sur le transfert et sur l'acquisition d'informations via la langue seconde, et non pas visant la langue elle-même au premier plan. Les principes de l'approche communicative sont respectés dans les programmes d'immersion, signalant l'échange des idées et l'emploi de la L2 dans des situations de communication. Il faut noter que les programmes immersifs canadiens sont ancrés dans le contenu scolaire transmis dans la L2, alors que la structure de la L2 n'est pas la cible principale de l'enseignement, du moins dans les classes supérieures. Le lexique est normalement enseigné dans le contexte de la lecture et du déroulement des activités scolaires traditionnelles routinières (projets, débats, etc.).

Quels sont les buts fondamentaux du programme d'IF ? D'après le Guide pédagogique (s.d.) du ministère de l'Éducation de la province du Nouveau-Brunswick (Canada; désormais N.-B.), son but ultime est de développer chez l'apprenant « la capacité de comprendre et d'utiliser la langue française comme outil de communication, d'interaction, de compréhension et d'expression dans une variété de situations orales et écrites » (p. 2). Ce mandat reflète les initiatives du gouvernement fédéral pour augmenter les niveaux de bilinguisme $^{\mathrm{i}}$ dans tout le Canada (Rehorick et al. 2006 : 1). Les architectes du programme immersif déclarent que les finissants " seront capables de comprendre, de parler, de lire et d'écrire une langue (seconde) [...] et de communiquer effectivement " ( $L$ 'art du langage $2003: 35$, nous soulignons). Pourtant l'objectif principal de compétence communicative est-il atteint ? Autrement dit, les élèves immersifs se montrent-ils compétents en L2 ? La réalité ne reflète pas cette aspiration.

Chaque année, le ministère de l'Éducation de la province entreprend l'évaluation des compétences en lecture et en rédaction des élèves de la maternelle à la $12^{\mathrm{e}}$ année, y compris de ceux et celles inscrits au programme d'IF. Des évaluations provinciales en lecture, en écriture et en performance orale en français sont administrées à un échantillon d'élèves immersifs de la $10^{\mathrm{e}}$ année ainsi qu'aux élèves de la $12^{\mathrm{e}}$. Les résultats récents de ces évaluations démontrent clairement que les élèves immersifs de la province du N.-B. éprouvent des difficultés de rendement, surtout en écriture en L2. Par exemple, au cours de l'année scolaire $2013-2014^{\mathrm{ii}}$, seulement $74 \%$ des élèves de la $10^{\mathrm{e}}$ année scolaire dans le District scolaire anglophone Est ont obtenu un score acceptable en lecture et seulement $41,63 \%$ de ce même groupe ont obtenu un score acceptable en écriture (Assessment 2014). Ces scores décevants ne sont pas uniquement attribuables à une école, à un programme ou à une seule année scolaire.

C'est dans les catégories du choix de mot, de la structure de la phrase et des conventions que les élèves éprouvent le plus de difficulté. Les exigences relatives à la catégorie " choix de mot » requièrent que l'élève puisse employer « un vocabulaire bien choisi qui rend le texte clair » et qu'il ait recourt à « un vocabulaire précis et bien choisi ou nuancé », ce qui lui permettra d'obtenir une note de "performance forte" dans les épreuves écrites (Framework 2013 : 28). De toute évidence, ces objectifs sont encore loin d'être atteints. 


\section{Lacunes constatées}

L'approche communicative valorise une orientation vers la fluidité orale, laissant de côté la précision grammaticale et lexicale requise pour une maîtrise linguistique authentique. Pourtant, un locuteur qui est incapable de construire un message compréhensible et bien structuré ne peut prétendre être doté de compétence communicative. Le fait d'accentuer la dimension orale de l'approche communicative minimise l'importance de l'étude lexicale de la L2. L'apprenant est effectivement éloigné d'une compétence réellement native. Les élèves immersifs n'ont généralement pas de contact continu ou étendu avec des locuteurs natifs du français (2016 Grade 12 Exit Survey). En ce qui concerne le lexique, l'introduction d'un vocabulaire de plus en plus riche et complexe dans un programme immersif n'est pas garantie : les contraintes de temps et de formation des enseignants, souvent eux-mêmes non-natifs, agissent négativement sur la réussite de l'approche communicative. L'imprégnation souhaitée est insuffisante. L'input est trop souvent lacunaire ou pire, fautif.

Il n'est pas raisonnable de s'attendre à un emploi sophistiqué et à une augmentation significative du vocabulaire des élèves sans qu'ils soient incités à creuser dans la richesse du système linguistique de la L2. L'approche communicative immersive semble ne pas donner lieu à la compétence lexicale souhaitée auprès des élèves, du moins de ceux de notre terrain. Il nous incombe de poser aussi la question de la disponibilité des outils visant l'acquisition du vocabulaire; en effet, nous avons constaté d'importantes lacunes sur le plan des éléments lexicaux présentés dans les divers recueils, manuels et autres ressources pédagogiques. De toute évidence, les carences lexicales sont multiples et contribuent chacune au déficit lexical constaté sur le terrain : absence d'outils prônant le lexique et de méthodes attestées pour l'enseignement du vocabulaire dans le contexte immersif décrit plus haut, manque de direction théorique dans l'enrichissement lexical des apprenants. Pourtant les recherches ont clairement établi que le vocabulaire est une matière fondamentale pour le développement des compétences linguistiques.

\section{Fondement théorique de l'expérimentation}

L'application des théories a toujours été le grand souci des praticiens. Depuis des siècles, les chercheurs et les pédagogues ont œuvré afin de trouver la façon la plus satisfaisante d'intégrer le lexique dans l'enseignement de la langue (Nunan 2015:8), car "les connaissances lexicales constituent l'une des composantes les plus importantes de l'acquisition du langage » (David $2000: 1$ ). Quelle est la meilleure façon d'amener les apprenants vers la compétence lexicale ?

L'enseignant qui voit au développement de la compétence linguistique de ses apprenants peut procéder de différentes façons. L'enseignement traditionnel de la L2 préconise la connaissance, l'exploration et la maîtrise des règles linguistiques (c'est-à-dire grammaticales) avant l'application pratique de celles-ci. Les approches traditionnelles d'enseignement sont alors de nature déductive et l'attention porte en priorité sur la langue et sur sa structure. Il s'agit alors d'une intervention directe (R. Ellis 2012 : 837) et c'est à l'apprenant d'appliquer les connaissances acquises à la production orale ou écrite subséquente. L'enseignement explicite et intentionné de la langue précise la structure et le contenu de celle-ci. Or, les tendances récentes en enseignement des langues préconisent une approche inductive, c'est-à-dire que l'attention porte plutôt sur la langue écrite ou orale telle qu'elle est observée et rencontrée lors des activités langagières. Une connaissance des règles grammaticales découle de la pratique et de l'observation de la langue "en jeu », c'est-à-dire quand elle est utilisée (Wilkins 1990). C'est en parlant, en entendant, en lisant ou en écrivant la langue que l'apprenant découvre sa structure interne. Notons que les 
approches inductives et déductives sont considérées toutes deux comme des approches explicites, c'est-à-dire intentionnelles, étant donné qu'une discussion des règles linguistiques accompagne ou suitiii le contact avec les items à apprendre dans chacune (Hulstijn 2005). Selon Hulstijn, l'intention est de découvrir le système, ses concepts et ses règles (p. 213). Il s'agit d'un procédé tout à fait conscient (N. Ellis $1994: 1)$ qui constitue des premiers pas vers le développement de la compétence métalinguistique. La grammaire et le vocabulaire sont discutés et analysés, soit avant ou pendant des leçons portant précisément sur la forme linguistique, soit en rencontrant et soulignant les traits linguistiques pendant la lecture. Comparons l'enseignement explicite avec l'apprentissage implicite, c'est-à-dire par incidence, lorsque l'apprenant acquiert des règles linguistiques par imprégnation simplement en participant à des activités langagières diverses (Read 2004 : 146) et sans qu'il en soit conscient ou qu'il cherche délibérément à découvrir ces règles. La différence entre l'implicite et l'explicite repose sur l'intention de l'apprenant et son niveau de conscience pendant l'activité. Il est éventuellement possible qu'une connaissance explicite des formes linguistiques devienne implicite à travers la répétition, le contact avec des locuteurs natifs dans des situations de communication naturelle, etc. (Gass et Selinker $2008: 243$ ).

Alors que certaines recherches démontrent l'efficacité de l'approche implicite, surtout pour les apprenants plus avancés, d'autres démontrent que le vocabulaire peut s'accroitre considérablement par l'apprentissage délibéré des mots (Nation 1990 ; Zimmerman 1997; Paribakht et Wesche 1997) ${ }^{\mathrm{iv}}$. L'enseignement explicite du vocabulaire implique que l'enseignant attire l'attention des élèves sur les mots eux-mêmes, à leur orthographe, à leurs définitions, à leurs dérivés, à leurs contenus morphologiques ainsi qu'à leurs conditions d'usage dans des phrases. Le mot lui-même est l'objet d'étude : sa forme, sa prononciation et son sens. L'apprenant se livre à des activités de décodage et de mémorisation du mot, c'est-à-dire à l'observation consciente des éléments «micro » du mot ${ }^{v}$. Pour ce qui est de la formule de présentation des mots aux apprenants de la L2 quel que soit leur âge, l'approche explicite de l'enseignement du vocabulaire prend couramment plusieurs formes. Dans certaines méthodes et approches d'enseignement traditionnelles, le vocabulaire est régulièrement enseigné au moyen de listes de mots isolés traduits ou pas dans la $\mathrm{L} 1$, de « chunks » ou des blocs lexicaux, ou encore à l'intérieur de dialogues, de scénettes ou de «situations » expérientielles. Quelle que soit la méthode ou l'approche, l'enseignement explicite du vocabulaire consiste à mémoriser des listes de mots, lesquels proviennent de textes à lire ou à traduire.

En tant qu'exercice de mémoire et en fonction de mécanisme organisateur, il est probable que les listes de mots ont un rôle important à jouer. Elles peuvent tenir lieu d'«outil cognitif et linguistique [...] à construire des catégories et des modes de référence » (Paveau 2006, cité dans Nonnan 2012 : 9). Toutefois, l'efficacité de l'enseignement de longues listes lexicales en contexte à partir d'un texte de lecture ou hors contexte via de simples listes de mots reste à prouver ${ }^{\text {vi }}$. D'ailleurs, le lexique d'une langue n'est pas une masse d'étiquettes de la réalité comme le prétend la " pédagogie de la liste » (Nonnan 2012 : 5). Il va de soi qu'on enseigne les mots pour aider à construire les connaissances linguistiques et extralinguistiques de l'apprenant.

Loin de la mémorisation de longues listes de mots isolés, Vancomelbeke (2004) présente plusieurs approches communes à l'apprentissage et à l'enseignement du vocabulaire du point de vue purement linguistique, à savoir 1) l'approche philologique, 2) l'approche syntaxique et 3) l'ensemble de perspectives visant l'analyse des mots comme signe (soit référentielle, morphologique et sémantique). L'objectif de chacune d'elles est l'étude explicite des mots. Des diverses approches à intérêt sémantique, Vancomelbeke souligne tout particulièrement l'approche actancielle comme approche modèle car elle réussit le 
mieux à mettre en valeur la relation de sens porté par le verbe et ses actants. D'après lui, l'approche actancielle reconnaît simultanément la place du mot dans son contexte communicatif, la structure syntagmatique nécessitée par le mot et le sens véhiculé par sa sélection parmi d'autres mots synonymiques. L'attention porte sur les fonctions grammaticales et significatives du mot choisi (2004:21), unissant ainsi la forme du mot et son (ou ses) sens. Cette approche valorise la reformulation syntaxique et sémantique, soit l'éventail de synonymes, d'antonymes, de collocations, de dérivations et d'expressions idiomatiques autour du mot choisi. Sur le plan sémantique, l'union de la syntaxe et de la sémantique dans le cadre d'un enseignement systématique du vocabulaire permet d'aménager une place au vocabulaire dans un programme généralement surchargé et dominé par la grammaire. Grâce à ces forces, l'approche actancielle semble combler les lacunes des autres approches linguistiques et constitue la meilleure option.

Il apparait ainsi que pour résoudre le problème des lacunes lexicales, il faut une méthode d'enseignement explicite du vocabulaire fondée sur une théorie sémantico-syntaxique claire et logique qui se transforme aisément en une approche didactique répondant aux besoins ressentis pour notre population cible.

\section{Le champ actanciel comme point de départ}

L'étude du vocabulaire par thèmes aboutit-elle à une connaissance linguistique approfondie, ou sert-elle plutôt à l'étiquetage des objets du monde ? L'activité lexicale se réduit à la dénomination des objets dans l'environnement de l'apprenant. Les listes générées, en fait des inventaires de noms, sont le plus souvent dépourvues de verbes qui sont le «moteur» de la phrase ${ }^{\text {vii }}$. Par exemple, l'étude du thème « corps humain » présente les mains comme une partie du corps à reconnaître et à nommer parmi plusieurs autres - les yeux, les épaules, les genoux et ainsi de suite. Le résultat est un accroissement des connaissances encyclopédiques des mots sur un thème, mais pas un accroissement des connaissances linguistiques touchant l'utilisation du mot dans le discours.

À l'inverse de l'étude lexicale thématisée, l'étude du vocabulaire par réseaux déclenche la " grosse machine sémantique » (Picoche et Rolland 2002:12) qui est la langue. Dans l'étude par réseaux, le mot main devient le tremplin pour l'étude linguistique des dérivés nominaux et verbaux comme manucure, manœuvre, mendiant, manipuler, l'étude des synonymes et des antonymes (on ouvre la main pour donner, la main fermée doigts serrés indique un poing; avoir les mains pleines / vides), des expressions figées et idiomatiques comme prendre en main, à portée de la main, mettre la main au feu, etc. Les idées de puissance (avoir les mains liées) et de force (taper du poing), de travail (un travail manuel) et d'admiration (applaudir, battre des mains) s'y entrelacent ${ }^{\text {viii }}$. Selon Picoche (ibid.),

[le] travail par réseaux aboutit à rendre compte de la polysémie du mot pris pour point de départ, qui n'est pas un fâcheux accident, mais un caractère fondamental du langage humain et de la cohérence sémantique interne du réseau qu'il commande. C'est le propre d'une conception linguistique de l'étude du lexique.

\section{Ainsi obtient-on un champ actanciel.}

Un des deux piliers de l'approche actancielle, le champ actanciel comprend l'ensemble de mots qui forment un réseau sémantique autour du mot central, ladite " constellation » de Picoche. Les mots qui se trouvent en proximité sémantique forment le champ actanciel ainsi que les mots reliés par la dérivation, tout autant pour les verbes que pour les noms 
(main) que nous venons d'illustrer. En fait, le champ actanciel croît au moyen de plusieurs procédés opérant sur les constituants du schéma actanciel de la phrase. Il s'agit de la nominalisation des actants, de la qualification des prédicats, de la dérivation morphologique et de la dérivation sémantique.

D'après Picoche, les divers aspects linguistiques de l'approche actancielle - synonymie et antonymie, ainsi que dérivation, polysémie et reformulation - sont essentiels à l'étude du lexique. Elle constate aussi l'utilité de l'approche actancielle dans l'enseignement lexical aux apprenants non francophones. Selon elle, l'enseignement des synonymes, des antonymes, et de la polysémie y compris la métonymie et la métaphore peut «faciliter grandement aux allophones la mémorisation des locutions figées dont l'importance est si grande dans les langues » (Picoche et Rolland $2002: 20$ ). Ces aspects se prêtent à une véritable exploration linguistique en profondeur chez l'apprenant.

Cependant, l'approche actancielle n'est pas simplement l'élaboration d'une théorie lexicale. Elle a été conçue pour l'application en salle de classe afin de promouvoir le développement de la compétence lexicale. L'application comporte deux composantes, à savoir les conventions d'enseignement proposées par Picoche et les outils lexicopédagogiques, soit Le Dictionnaire du français usuel (2002) également disponible en version électronique (DFUi), et le plus récent ouvrage didactique Vocalire (2012), qui est une version condensée et " améliorée " ${ }^{\mathrm{ix}}$ du $D F U$ traitant de 7500 mots. Ce sont ces outils qui ont servis à élaborer les leçons de vocabulaire français utilisées sur notre terrain.

\section{Développement du matériel pédagogique}

Dans son document Le vocabulaire et son enseignement (Germain et Picoche 2013), Picoche détaille le protocole de mise en œuvre pour l'application de l'approche actancielle en salle de classe. Cette mise en pratique repose sur quatre principes fondamentaux. Premièrement, on donne la priorité au verbe, puisque le verbe est central au sens de la phrase, et le choix du sujet et des compléments s'organise toujours autour de lui. Le sens du verbe est alors contextualisé. Deuxièmement, on doit associer l'étude de la syntaxe à celle du vocabulaire, c'est-à-dire que le mot doit apparaître dans une phrase illustrative pour montrer sa place dans la structure syntaxique. Troisièmement, il faut partir du mot et non de la chose, pour éviter le simple étiquetage des objets dans l'environnement de l'élève et pour encourager l'étude linguistique du mot et de sa constellation. Il faut en même temps s'assurer d'employer une terminologie linguistique (nominaliser les composants linguistiques tels que substantif, adjectif, préfixe, etc.) surtout dans les classes scolaires supérieures. Finalement, il faut partir de mots simples, déjà acquis par les élèves, universellement connus par les francophones, des mots fréquents, usuels, enfin des mots «bien connus et convenablement manipulés » (Germain et Picoche 2013 : 6). Cette approche permet de bâtir sur le « déjà su » pour viser le nouveau.

Pour ce qui est du dispositif d'enseignement, Picoche recommande quatre séances pour l'étude d'un mot. La première séance d'enseignement du vocabulaire se consacre à la présentation du mot déclencheur (qu'elle appelle mot pilote, Picoche et Rolland $2012: 6$ ) et au remue-méninge qui fait ressortir les mots associés au mot pilote. Pour un nom, on identifie les adjectifs qui qualifient ce nom. Pour un verbe, on imagine des sujets, des objets, des compléments possibles. Les leçons subséquentes concernent l'exploitation linguistique de ce mot, au moyen de la création de phrases contenant le mot pilote ainsi que par la reformulation de celles-ci au moyen de la dérivation et de la synonymie. L'ajout des expressions figurées ou idiomatiques et une discussion du niveau de formalité (c'est-à-dire $\mathrm{du}$ registre) s'ensuivent. La rédaction d'un texte sur un thème relié au mot pilote et l'analyse des textes écrits par les élèves terminent l'étude. S'enchaîne naturellement l'étude 
linguistique des mots qui sont ressortis de cette première leçon. Autrement, l'enseignant peut choisir un nouveau mot non connecté au premier, et le processus d'apprentissage du vocabulaire recommence.

Pour pallier l'absence de matériel pédagogique pour l'enseignement explicite du vocabulaire, notre première tâche a été de créer une trousse de matériel pédagogique en utilisant les ouvrages lexico-pédagogiques de Rolland et Picoche. Nous avons examiné les articles de ces deux ouvrages pour trouver les éléments lexicaux à mettre au programme. Notre souci principal était de mettre en valeur tout ce qui éveillerait une conscience des aspects morphologiques, syntaxiques et sémantiques du verbe à l'étude.

Pour ancrer nos leçons, nous avons choisi le verbe DIRE, le «verbe de communication » par excellence. Selon Dubois et Dubois-Charlier (1997), les verbes de communication comprennent tous les verbes qui ont trait à la transmission (d'idées, de discours ou de sentiments) par des cris, des paroles ou des sons, ainsi que les notions d'interpeller, d'informer, de demander et d'ordonner, d'interroger et de prier. Le verbe DIRE s'emploie pour exprimer oralement des pensées intérieures. Il est aussi résomptif de nature, permettant la récapitulation des idées déjà exprimées. Ses synonymes comprennent les verbes 'directs' (ÉNONCER, PROMETTRE, ARTICULER, EXPRIMER, etc.) ainsi que les verbes pour reprendre ou pour résumer la parole d'un autre (RACONTER, REDIRE, RÉPÉTER, RAPPORTER, etc.). De plus, les verbes de communication, dits parfois verbes de parole, sont très intéressants au niveau syntaxique de par leurs compléments nominaux ainsi qu'au niveau sémantique dans leur rôle performatif (Giry-Schneider 1994 : 103). La grande constellation de synonymes du verbe nous a ainsi guidée dans le choix du verbe DIRE. En outre, l'étude du verbe DIRE s'ancre très naturellement dans les objectifs communicatifs du programme du français immersif, dont la sensibilisation des élèves à la compétence communicative.

Le contenu retenu pour les leçons a été présenté dans les leçons selon la logique du $D F U$ qui commence par l'acception la plus usuelle pour passer ensuite aux sens plus abstraits puis idiomatiques du verbe. Le tableau 1 met en correspondance le contenu d'une des leçons de vocabulaire (Leçon 4) et la section de l'article de base :

Tableau 1. Contenu d'une leçon sur le verbe DIRE

\begin{tabular}{|c|c|c|c|c|}
\hline $\begin{array}{l}\mathrm{N}^{0} \mathrm{de} \\
\text { la } \\
\text { leçon }\end{array}$ & $D F U$ & Intérêt lexical & Contenu lexical & $\begin{array}{c}\text { Terminologie } \\
\text { grammaticale ou } \\
\text { actancielle }\end{array}$ \\
\hline \multirow[t]{2}{*}{4} & \multirow[t]{2}{*}{$\begin{array}{l}\text { Sections } \\
\text { II et III }\end{array}$} & Synonymes & $\begin{array}{l}\text { ANNONCER, ÉNONCER, } \\
\text { DÉNONCER, REDIRE, } \\
\text { RÉPÉTER, CONTREDIRE, } \\
\text { MÉDIRE de, DICTER, SE DIRE, } \\
\text { PRONONCER, RÉCITER, } \\
\text { contradiction, contradictoire, } \\
\text { diction, prononciation, } \\
\text { énonciation, énoncé, récitation }\end{array}$ & \multirow[t]{2}{*}{ négatif, infinitif } \\
\hline & & $\begin{array}{c}\text { Contenu } \\
\text { sociolinguistique } \\
\text { (le statut social) }\end{array}$ & $\begin{array}{l}\text { ORDONNER, DEMANDER, } \\
\text { CONSEILLER, } \\
\text { ordre, conseil }\end{array}$ & \\
\hline
\end{tabular}

Selon la correspondance avec les sections de l'article du DFU (colonne 2), sont inclus l'intérêt lexical principal de la leçon (colonne 3) et son contenu lexical (colonne 4). Il s'agit 
ici du « nouveau vocabulaire » qui est l'objet de l'enseignement explicite du vocabulaire et des apprentissages des élèves, lequel est évalué lors du test de vocabulaire. Notons que les verbes sont en majuscule, tandis que les noms, les adjectifs, etc. sont en minuscule et en italique. La dernière colonne (colonne 5) fournit la terminologie grammaticale utilisée dans la leçon. En somme, chaque leçon présente aux élèves entre 3 et 14 verbes sémantiquement liés au mot pilote DIRE, et entre 7 et 12 noms, adjectifs et adverbes dérivés des verbes ou liés à ces verbes. Le tableau complet se trouve en annexe.

La sélection des contenus linguistiques étant faite, nous avons développé cinq types de matériels pour chaque leçon, à savoir 1) des fiches pédagogiques, souvent appelées plans de cours, qui décrivent le déroulement de la leçon pour guider l'enseignant, 2) des présentations du contenu dans des diaporamas (PowerPoint), 3) des fiches de travail que les élèves remplissent en cours de leçon ${ }^{\mathrm{x}}$ et 4 ) des activités de réinvestissement ${ }^{\mathrm{xi}}$. Nous avons également préparé 5) une fiche technique qui explique le système actanciel aux enseignants.

Chaque leçon de vocabulaire durait 60 minutes et toutes avaient le même format. Nous avons consacré le début de chaque séance d'enseignement au quiz hebdomadaire qui rappelait le contenu de la leçon précédente et qui devait être complété en cinq minutes environ. Venait ensuite la discussion du mot cible qui consistait en une exploration du mot sur les axes paradigmatique et syntagmatique. Cet échange durait environ 30 minutes. Il était suivi de l'activité de réinvestissement qui durait environ 30 minutes, selon le temps disponible.

\section{Evaluation du développement de la compétence lexicale}

\subsection{Le test de vocabulaire}

Notre expérimentation a été conduite dans deux classes de français L2 auprès d'élèves âgés de 16 et 17 ans. Les deux classes d'élèves qui ont participé à l'expérimentation étaient composées chacune de 29 élèves, soit un total de 58 élèves. Dans le but de mieux vérifier l'effet de l'enseignement explicite du vocabulaire sur le groupe expérimental, nous avons mené le même test de vocabulaire auprès d'un groupe contrôle de 27 élèves du même niveau scolaire et de la même école.

Afin d'évaluer les acquis lexicaux et de pouvoir comparer les connaissances lexicales des élèves avant et après l'enseignement explicite du vocabulaire, nous avons développé un test de vocabulaire que nous avons administré à trois reprises. Ce test à trous incorporait les mots de la constellation du verbe DIRE présentés aux élèves tout au long des leçons. Voici quelques exemples des phrases du test :

1. Quelle est la s de l'expression « J'ai un mot à vous dire $» ?$

2. Paul aime bien son ami Stéphane mais il n'aime pas Jean-Pierre. Paul dit du bien de Stéphane mais il $\mathbf{m}$ toujours de Jean-Pierre.

3. Quelle heure est-il ? L'horloge nous d

4. Le président de la République fait une $\mathbf{d}$ l'heure. guerre contre ses ennemis.

La plupart des phrases du test ont été construites en se servant des exemples tirés des différentes sections du $D F U$ et de Vocalire. Le même test a été administré aux trois séances d'évaluation, d'abord à la première rencontre avec les élèves, c'est-à-dire avant la première 
leçon de vocabulaire, pour évaluer l'état des connaissances des élèves avant l'expérimentation; il s'agit du «Pré-test». Le test a également eu lieu à la fin des leçons («Post-test $1 »)$ pour évaluer les apprentissages, et une dernière fois cinq semaines après la dernière rencontre avec les élèves («Post-test $2 »)$. L'objectif de l'évaluation différée était de voir si les mots avaient été retenus même après une période de temps sans retour sur la matière.

À la lumière des différentes définitions de connaissances lexicales vues à la section 1 , le test de vocabulaire visait principalement l'évaluation de la " connaissance du vocabulaire et la capacité de l'utiliser» (Cadre 2001 : 4.7.2.1.1), même hors d'un contexte "communicatif» (compris comme oral). Les élèves ont été amenés à faire la preuve de leurs connaissances des formes écrites des mots et de leurs constituants morphosyntaxiques et des sens des mots. De plus, ils ont été amenés à démontrer leur compétence lexicale productive (au moyen de l'écrit), tout en recherchant et extrayant de leur lexique mental (Singleton 1999) les mots requis pour réussir le test. Soulignons que les 20 phrases du test étaient entièrement fondées sur l'article du $D F U$, même si les formulations précises ont parfois été forgées par la chercheuse.

\subsection{Résultats des tests de vocabulaire}

L'amélioration globale du groupe expérimental entier a été constatée dans la progression des scores au fil des trois épreuves (Pré-test, Post-test 1, Post-test 2). Les scores globaux entre le Pré-test et le Post-test 1 montrent une progression de plus de 3 points par élève sur les 20 points disponibles. De même, entre le Post-test 1 et le Post-test 2, le score total s'est amélioré davantage en passant à 0,40 points de plus par élève. L'augmentation totale des scores entre le Pré-test et le Post-test 2 était donc de 3,53 points par élève. Pour le groupe contrôle, les scores globaux entre le Pré-test et le Post-test 1 ont connu une petite amélioration de 0,58 point par élève. Par contre, les scores du groupe contrôle ont baissé entre le Post-test 1 et le Post-test 2 : là, le score a diminué de 0,14 point par élève. Ainsi le pourcentage d'amélioration des élèves du groupe expérimental est de 17,7\% [3,53 points en moyenne par élève $/ 20$ points], tandis que celui des élèves du groupe contrôle est seulement de $2,2 \%$ [0,44 points en moyenne par élève/20 points].

Les résultats globaux montrent une évolution significative des scores du groupe expérimental à chaque étape d'évaluation. Les élèves ont pu fournir les mots exigés pour les phrases données de façon croissante en moyenne à chaque épreuve. Il semble que l'enseignement explicite du vocabulaire ait porté fruit. Aussi, le fait d'avoir obtenu de meilleurs résultats cinq semaines après la fin de l'expérimentation au Post-test 2, et sans intervention pédagogique aucune entre le Post-test 1 et le Post-test 2, est indicateur de résultats intéressants reliés à l'enseignement explicite du vocabulaire.

\section{Bilan}

Les résultats des évaluations sur le plan du développement des compétences lexicales indiquent que la méthode d'enseignement explicite du vocabulaire selon l'approche actancielle a eu des effets positifs. Dans un sondage à la fin de l'expérimentation ${ }^{\text {xii }}$, l'enseignante titulaire s'est dite ravie du programme d'enseignement. Elle était très satisfaite de la nature systématique des leçons et elle a exprimé le désir de poursuivre l'enseignement explicite du vocabulaire avec ses élèves. L'appréciation globale des élèves 
sur les leçons ${ }^{\mathrm{xii}}$ a également été positive. Ils ont aimé apprendre les nouveaux mots et ont trouvé les leçons assez utiles. De nombreux élèves ont fait un usage fructueux des fiches de travail, tout autant pour suivre la progression des leçons que pour en réviser le contenu d'une semaine à l'autre. Les activités de réinvestissement faites en dyades ou en groupes leur ont aussi généralement plu. De nombreux élèves ont mentionné le plaisir d'apprendre le vocabulaire français et ils ont eu la nette impression d'avoir élargi leur répertoire lexical. Plusieurs ont dit se sentir plus sûrs d'eux quand ils employaient les nouveaux mots et pour d'autres, c'est l'expression écrite qui s'est améliorée, grâce au nouveau bagage de mots plus sophistiqués.

Quant au matériel lexicographique ( $D F U$ et Vocalire) qui a servi de base du matériel pédagogique, nous estimons que la progression logique de leur présentation des mots a contribué grandement au développement des leçons et à la composition du test de vocabulaire. La division de l'article dictionnairique en acceptions des plus concrètes aux plus abstraites, clairement numérotées et introduites par une phrase ordinaire et accessible, a permis d'identifier assez rapidement l'acception voulue, ce qui a contribué à sa bonne utilisation.

\section{Conclusion}

Si les élèves ont fait preuve convaincante d'une augmentation de la compétence lexicale dans le contexte de notre étude, nous pouvons dire que l'enseignement explicite du vocabulaire, mis en marche au moyen de l'application d'une approche syntacticosémantique solide et des outils lexico-pédagogiques riches, a réussi à mener les élèves à l'apprentissage et à la rétention de nouveaux mots. Nous espérons qu'une expansion future de l'expérimentation contribuera davantage à ce développement critique de la compétence lexicale chez des élèves immersifs.

\section{Références bibliographiques}

L'art du langage. (2003). Fredericton, Nouveau-Brunswick : Ministère de l'Éducation du NouveauBrunswick.

Assessment 2014. (2014) Texte consulté en ligne à l'adresse suivante : http://web1.nbed.nb.ca/sites/ASD-S/Documents/20142015\%20Assessment\%20DEC\%20Presentation.pdf

Bassano, D. (1998). L'élaboration du lexique précoce chez l'enfant français: structure et variabilité. Enfance, 51(4), 123-153.

Cadre européen commun de référence pour les langues (CECR). (2001). Conseil de l'Europe. Strasbourg: Unité des Politiques linguistiques. Texte consulté en ligne à l'adresse suivante : http://www.coe.int/T/DG4/Linguistic/Source/Framework FR.pdf

Chevalier, G. (2013). L'enseignement du vocabulaire: la grammaire du mot et l'approche actancielle. Notes de cours non publiées. Moncton N.-B. : Université de Moncton.

Coady, J. (1997). L2 vocabulary acquisition through extensive reading. Dans Coady, J. \& Huckin, T. (eds). Second Language Vocabulary Acquisition. Cambridge: Cambridge University Press, 225-237.

Coady, J. \& Huckin, T., eds. (1997). Second Language Vocabulary Acquisition. Cambridge: Cambridge University Press.

David, J. (2000). Le lexique et son acquisition: aspects cognitifs et linguistiques. Le Français aujourd'hui, 131, 31-41.

Dubois, J. \& Dubois-Charlier, F. (1997). Les verbes français. Paris : Larousse-Bordas.

Ellis, N. (1994). Consciousness in second language learning: Psychological perspectives on the role of conscious processes in vocabulary acquisition. Consciousness in second language learning AILA Review 11. 37-56.

Ellis, R. (2012). Language teaching research and language pedagogy. Malden, Mass.: John Wiley \& Sons. 
Folse, K.S. (2004). Myths about teaching and learning second language vocabulary: What recent research says. TESL Reporter 37(2), 1-13.

Framework for Provincial Assessments. (2013). Ministère de l'Éducation, Province du NouveauBrunswick. Fredericton N.-B. Texte consulté en ligne à l'adresse suivante : http://www.gnb.ca/0000/results/pdf/AssessmentFrameworkDocument.pdf

Gass, S.M. \& Selinker, L. (2008). Second language acquisition: An introductory course (2nd ed.). Mahwah, NJ: Lawrence Erlbaum Associates.

Germain, B. \& Picoche, J. (2013). Le vocabulaire et son enseignement : Présentation. Disponible à Vocanet : www.vocanet.fr. Texte consulté en ligne.

Giry-Schneider, J. (1994). Les compléments nominaux des verbes de parole. Langages, 28(115), 103125.

Guide pédagogique. (Sans date). Document No 843420. Fredericton, Nouveau-Brunswick : Ministère de l'Éducation du Nouveau-Brunswick.

Harley, B. \& Jean, G. (1999). Vocabulary skills of French immersion students in their second language. Zeitschrift für Interkulturellen Fremdsprachenunterricht 4(2). Texte consulté en ligne le 2 novembre 2013 à l'adresse suivante : www.spz.tudarmstadt.de/projekt_ejournal/jg-04-2/beitrag/harley2.htm.

Harmer, J. (2015). The practice of English language teaching. New York: Pearson.

Hulstijn, J. (2005). Theoretical and empirical issues in the study of implicit and explicit secondlanguage learning: Introduction. Studies in second language acquisition, 27(2), 129-140.

Jared, D. (2008). Les évaluations à grande échelle de l'alphabétisation des élèves en immersion française. Encyclopédie sur le développement des jeunes, 1-9. London, On.: Réseau canadien de recherche sur la langue et l'alphabétisation. Texte consulté en ligne à l'adresse suivante : http://www.literacyencyclopedia.ca/pdfs/topic/php?topld=239\&fr=true

Jiang, N. (2000). Lexical representation and development in a second language. Applied linguistics, 21(1), 47-77.

Nation, P. (1990). Teaching and learning vocabulary. New York: Newbury House.

Nonnan, É. (2012). La didactique du français et l'enseignement du vocabulaire, dans vingt ans de revues de didactique du français langue première. Repères 46, 33-72.

Nunan, D. (2015). Teaching English to speakers of other languages: An introduction. New York: Routledge.

Paribakht, T.S. \& Wesche, M. (1997). Vocabulary enhancement activities and reading for meaning in second language vocabulary acquisition. Dans J. Coady \& T. Huckin (Eds.), Second language vocabulary acquisition: A rationale for pedagogy. Cambridge: Cambridge University Press, 174-199.

Paveau, M.-A. (2006). Leçon de vocabulaire 1. Le français d'aujourd'hui, 154, 121-128.

Picoche, J. (1992). Précis de lexicologie française: l'étude et l'enseignement du vocabulaire, $2^{\mathrm{e}}$ édition. (Vol. 18). Paris: Éditions Nathan. . (1993). Didactique du vocabulaire français. Paris: Éditions Nathan.

Picoche, J. \& Rolland, J.-C. (2002). Dictionnaire du français usuel: 15000 mots utiles en 442 articles. Bruxelles : De Boeck, Duculot.

Qian, D.D. (1996). ESL Vocabulary Acquisition: Contextualization and Decontextualization. The Canadian Modern Language Review / La revue canadienne des langues vivantes, 53(1), $120-142$.

Read, J. (2000). Assessing Vocabulary. Cambridge: Cambridge University Press. . (2004). Plumbing the depths: How should the construct of vocabulary knowledge be defined. Vocabulary in a second language, 209-227.

Rehorick, S., Dicks, J., Kristmanson, K. \& Cogswell, F. (2006). Quality Learning in French Second Language in New Brunswick. Fredericton: Second Language Learning Centre, University of New Brunswick.

Robinson, P.J. (1989). A rich view of lexical competence. ELT Journal 43(4), 274-282.

Rolland, J.-C. \& Picoche, J. (2012). Vocalire: Les 7500 mots essentiels du lexique français. Raleigh, North Carolina: Éditions Lulu.

Schmitt, N. (1998). Tracking the incremental acquisition of second language vocabulary: A longitudinal study. Language learning, 48(2), 281-317.

SIL. (1998). Consulté en ligne le $12 / 14 / 2015$ à l'adresse suivante : www01.sil.org/lingualinks/languagelearning.

Singleton, D.M. (1999). Exploring the second language mental lexicon. Ernst Klett Sprachen. 
Sökmen, A. (1997). Current trends in teaching second language vocabulary. Dans Schmitt \& McCarthy Vocabulary: Description, acquisition and pedagogy. Cambridge University Press, 237-257.

Sun, R., Mathews, R.C. \& Lane, S.M. (2007). Implicit and explicit processes in the development of cognitive skills: A theoretical interpretation with some practical implications for science education. Educational Psychology Research Focus, 1-26.

Tremblay, O. (2009). Une ontologie des savoirs lexicologiques pour l'élaboration d'un module de cours en didactique du lexique. Thèse doctorale. Université de Montréal.

Tréville, M.-C. (1987). Faut-il enseigner le vocabulaire dans la langue seconde ? Dans LeBlanc, R., Compain, J., Duquette, L. \& Séguin, H. (eds.). 1987. L'enseignement des langues secondes aux adultes: Recherches et pratiques. Ottawa: Les Presses de l'Université d'Ottawa, 159-182.

Tréville, M.-C. \& Duquette, L. (1996). Enseigner le vocabulaire en classe de langue. Paris: Hachette.

Turnbull, M., Lapkin, S., Hart, D. \& Lapkin, S. (1998). Time on task and immersion graduates' French proficiency. French second language education in Canada: Empirical studies. Toronto: University of Toronto Press, 31-55.

Vancomelbeke, P. (2004). Enseigner le vocabulaire. Paris : Éditions Nathan.

Wilkins, D.A. (1990). Second languages: How they are learned and taught. In N.E. Collinge (ed.) (1990) An Encyclopaedia of Language, 518-550. London: Routledge.

Zimmerman, C.B. (1997). Historical trends in second language vocabulary instruction. Dans Coady, J. \& Huckin, T. (eds.) Second Vocabulary Language Acquisition: A Rationale for Pedagogy. Cambridge: Cambridge University Press, 5-20.

2016 Grade 12 Exit Survey: What's on your mind? Anglophone Sector. (2016). Department of Education and Early Childhood Development. Province of New Brunswick, Fredericton N.B.

\section{Notes}

Cet article fut fondé principalement sur la thèse doctorale non publiée de l'auteure, Outiller l'immersion française pour l'enseignement explicite $d u$ vocabulaire au secondaire. Université de Moncton 2017.

'Mentionnons que le «bilinguisme» des élèves immersifs n'est pas un but déclaré du ministère de l'Éducation, mais vise plutôt la capacité de «communiquer » en français.

${ }^{i i}$ Les résultats pour l'année scolaire 2013-2014 sont les plus récents disponibles auprès du ministère de l'Éducation du N.-B.

iii Ou précède, dans le cas de l'enseignement déductif.

${ }^{\text {iv }}$ L'enseignement explicite entraîne non seulement une quantité supérieure de mots appris, mais aussi l'étendue de ceux-ci, selon des recherches de Paribakht et Wesche 1997 ; voir aussi Coady et Huckin 1997.

v Comparons ce processus avec la «macro », dans laquelle la langue est analysée globalement sans aucune attention particulière sur les éléments linguistiques spécifiques (Sun, Mathews et Lane 2007 ; Sökmen 1997). Harmer $(2015: 302)$ emploie une métaphore de la forêt: le processus d'analyse «micro» se penche sur toute la forêt, tandis que la «macro» examine chaque arbre individuellement.

vi De même, Qian (1996) a démontré qu'une liste de mots décontextualisés s'apprenait plus facilement qu'une liste contextualisée.

vii Selon David $(2000: 3)$, «c'est le verbe qui porte la plupart des informations sémantique et pragmatique ». Fait intéressant, d'après une étude de Bassano (1998), des enfants francophones de 20 mois employaient plus de verbes de plus que leurs paires anglophones dans une proportion de $18 \%$.

viii Exemples tirés du DFU MAIN, pp. 517 à 521.

${ }^{\text {ix }}$ Rolland 2017, communication personnelle. 


\begin{abstract}
${ }^{x}$ Parallèlement à la discussion, les élèves ont été amenés à prendre en note les informations importantes de la leçon - les mots déclencheurs, les actants, les synonymes, les schémas, etc.

${ }^{x i}$ Selon le protocole d'enseignement du lexique de Picoche et ses collègues, une activité de réinvestissement écrite est importante pour la consolidation des acquis lexicaux. Ainsi, à la suite de la discussion autour du verbe, les élèves étaient amenés à essayer les activités de réinvestissement développées pour favoriser cette consolidation des savoirs. Chaque activité de réinvestissement - la rédaction d'un court paragraphe écrit, par exemple, ou d'un dialogue inventé - incorporait le verbe déclencheur et son champ actanciel pour permettre une intégration immédiate des savoirs nouvellement acquis.

xii Parmi les 9 questions posées à l'enseignante étaient les suivantes: Est-ce que les leçons de vocabulaire étaient utiles pour vos élèves ? Si Oui, comment ? Si Non, selon vous, pourquoi ? L'objectif de l'étude était d'expérimenter une nouvelle méthode d'enseignement du vocabulaire pour l'amélioration des compétences lexicales des élèves immersifs. À votre avis, est-ce que cet objectif a été accompli ?

xiii Deux exemples des questions fournies aux élèves: Have you used the verb DIRE or any of its synonyms outside the vocabulary lessons (for example, in other writing assignments not specifically connected with the lessons, in conversations, at your job with coworkers, etc.)? Where, when and how? Please elaborate. The object of the study was to experiment a new method of enriching Immersion students' French vocabulary. In your opinion, how well was that objective met?
\end{abstract}

\title{
Annexe: Tableau 2. Les 5 leçons sur le verbe DIRE
}

\begin{tabular}{|c|c|c|c|c|}
\hline $\begin{array}{l}\mathbf{N}^{0} \mathrm{de} \\
\text { la } \\
\text { leçon }\end{array}$ & $D F U$ & Intérêt lexical & Contenu lexical & $\begin{array}{c}\text { Terminologie } \\
\text { grammaticale ou } \\
\text { actancielle }\end{array}$ \\
\hline 1 & & $\begin{array}{l}\text { Introduction: } \\
\text { L'approche } \\
\text { actancielle }\end{array}$ & & $\begin{array}{c}\text { actant, sens } \\
\text { figuré, tournure, } \\
\text { locution, } \\
\text { synonyme }\end{array}$ \\
\hline 2 & $\begin{array}{l}\text { Sections } \\
\text { I et II }\end{array}$ & $\begin{array}{l}\text { Le discours } \\
\text { direct } \\
\text { et indirect }\end{array}$ & $\begin{array}{l}\text { discours, sous-entendu, } \\
\text { conversation, RACONTER, } \\
\text { EXPLICITER, implicite, } \\
\text { FAIRE ALLUSION, } \\
\text { INSINUER, }\end{array}$ & $\begin{array}{l}\text { discours direct, } \\
\text { interjection, } \\
\text { guillemets, } \\
\text { discours indirect, } \\
\text { changements } \\
\text { syntaxiques, } \\
\text { passif, première } \\
\text { personne, } \\
\text { indicatif, pronom }\end{array}$ \\
\hline 3 & $\begin{array}{l}\text { Section } \\
\text { II }\end{array}$ & ANNONCER & $\begin{array}{c}\text { ANNONCER, PRÉVOIR, } \\
\text { PRÉVENIR, } \\
\text { signe annonciateur, AVERTIR, } \\
\text { DÉCLARER, déclaration, } \\
\text { les petites annonces, } \\
\text { prédiction, avertissement, } \\
\text { annonciation, annonciateur, } \\
\text { annonce (n.) }\end{array}$ & $\begin{array}{c}\text { reformulation, } \\
\text { nominal }\end{array}$ \\
\hline
\end{tabular}




\begin{tabular}{|c|c|c|c|c|}
\hline 4 & $\begin{array}{l}\text { Sections } \\
\text { II et III }\end{array}$ & $\begin{array}{l}\text { Contenu } \\
\text { sociolinguistique } \\
\text { (le statut social) }\end{array}$ & $\begin{array}{c}\text { ANNONCER, ÉNONCER, } \\
\text { DÉNONCER, REDIRE, } \\
\text { RÉPÉTER, CONTREDIRE, } \\
\text { MÉDIRE de, DICTER, SE DIRE, } \\
\text { PRONONCER, RÉCITER, } \\
\text { contradiction, contradictoire, } \\
\text { diction, prononciation, } \\
\text { énonciation, énoncé, récitation; } \\
\text { ORDONNER, DEMANDER, } \\
\text { CONSEILLER, } \\
\text { ordre, conseil }\end{array}$ & négatif, infinitif \\
\hline 5 & $\begin{array}{l}\text { Sections } \\
\text { V et VI }\end{array}$ & $\begin{array}{c}\text { les emplois } \\
\text { idiomatiques de } \\
\text { DIRE }\end{array}$ & $\begin{array}{l}\text { FAIRE COMPRENDRE, } \\
\text { SIGNIFIER, signification, } \\
\text { VOULOIR DIRE, PLAIRE (ne } \\
\text { rien DIRE) }\end{array}$ & \\
\hline
\end{tabular}

